Archives de sciences sociales des religions

176 | octobre-décembre 2016

Bulletin Bibliographique

\title{
Les traditions nationales en sociologie des religions
}

\section{Pierre Lassave}

\section{OpenEdition}

Journals

Édition électronique

URL : http://journals.openedition.org/assr/28142

DOI : 10.4000/assr.28142

ISSN : $1777-5825$

Éditeur

Éditions de l'EHESS

Édition imprimée

Date de publication : 31 décembre 2016

Pagination : 177-192

ISSN : 0335-5985

\section{Référence électronique}

Pierre Lassave, "Les traditions nationales en sociologie des religions ", Archives de sciences sociales des religions [En ligne], 176 | octobre-décembre 2016, mis en ligne le 01 janvier 2019, consulté le 04 janvier 2020. URL : http://journals.openedition.org/assr/28142 ; DOI : 10.4000/assr.28142 


\title{
Pierre Lassave
}

\section{Les traditions nationales en sociologie des religions}

\author{
À propos de : \\ Blasi Anthony J., GioRdan Giuseppe (éds.), Sociologies of Religion. \\ National Traditions, Leiden-Boston, Brill, coll. « Religion \& Social \\ Order ", 2015, 412 p. ${ }^{1}$
}

Sous réserve d'inventaire, il se pourrait bien que ce collectif soit le premier à réunir un ensemble d'histoires nationales de sociologies des religions de par le monde ${ }^{2}$. Les manuels, handbooks ou textbooks de cette spécialité disciplinaire présentent certes depuis des décennies théories et enquêtes dans divers cadres nationaux. Mais ici c'est le travail systématique de mise en contexte qui justifie l'alignement bout à bout de plusieurs pays. Entreprise apparemment peu orthodoxe si l'on considère que toute science tend par nature à se défaire de tout cadre institutionnel. Ne pas confondre en effet «traditions sociologiques» qui se jouent précisément des frontières nationales et « traditions nationales de sociologie » où la discipline se rappelle à sa condition historique. Les premières ont déjà fait l'objet d'essais classiques (Nisbet, Collins) alors que les secondes ne s'appréhendent que très épisodiquement ${ }^{3}$. L'histoire des savoirs nous a en effet rendu toujours plus attentifs à ce qui les construit pratiquement et les distinguent les uns des autres. Dans nos domaines d'objet religieux, la philosophie oppose ainsi communément la phénoménologie allemande au pragmatisme américain ou la théorie française à l'empirisme anglo-saxon. William H. Swatos qui préface l'ouvrage ajoute que l'existence même de ce recueil pionnier atteste que la sociologie des religions est un corps de savoir qui a désormais fait sa place dans le monde académique.

1. Voir sommaire en annexe. Je remercie Anne-Sophie Lamine et Paul-André Turcotte pour la relecture attentive de cette note critique et leurs remarques utiles.

2. Signalons cependant une tentative précédente, mais plus thématique, émanant de la Société internationale de sociologie des religions (SISR) : Sociology and Religions. An Ambiguous Relationship (L. Voyé, J. Billiet, éds.), Leuven, Leuven University Press, 1999.

3. Dans le premier registre : R. Nisbet, The Sociological Tradition, New York, Basic Books Publishers, 1966; R. Collins, Three Sociological Traditions, New York, Oxford University Press, 1985. Dans le second : La sociologie au XXe siècle, tome II (G. Gurvitch, W. E. Moore, dir.), Paris, Presses universitaires de France, 1947 ; National Traditions in Sociology (N. Guenov, éd.), London, Sage Publications, 1989. 
C'est en effet du réseau international d'enseignants-chercheurs noué par la plus ancienne association de sociologie des religions, qui a son siège aux ÉtatsUnis (Association for the Sociology of Religion), qu'est issu l'échantillon de quatorze pays retenus ${ }^{4}$. D'abord, bien évidemment, les sociétés fondatrices de la discipline générale (France, Allemagne, États-Unis, Grande-Bretagne), puis ses premières aires de diffusion (Belgique, Québec, Brésil, Italie) et ses espaces académiques progressivement enrôlés (Espagne, Pays-Bas, Canada anglais, Australie, Japon, Croatie). L'ensemble ne couvre pas le quart de la population mondiale ce qui indique les limites de l'entreprise. Le continent africain, la Chine, la Russie et l'Inde, sans parler du Proche-Orient, font ici cruellement défaut. En outre, les critères qui ont présidé à cette sélection de pays restent dans l'ombre, même si l'on peut deviner le rôle décisif joué par les relations d'interconnaissance entre universitaires américains. Mais un objectif ambitieux est affiché d'entrée de jeu par l'un des deux coordinateurs, Giuseppe Giordan de l'Université de Padoue : inviter à la réflexion sur les styles nationaux de recherche à partir des relations entre la spécialité disciplinaire et le cadre culturel plutôt que de dresser un catalogue des théories, des auteurs et des institutions.

Bien que n'appartenant pas à l'association en question, bien que n'ayant pas le profil du professeur de sociologie des religions, je me suis retrouvé dans ce navire pour présenter le singulier cas français. La proposition qui m'a été faite provient sans doute de l'absence momentanée de candidat pro forma, de mes contributions antérieures à la question ${ }^{5}$, et de relations entretenues depuis quelques années et de façon très indirecte avec quelques spécialistes du milieu. En toute rigueur académique, je devrais m'abstenir de commenter ce collectif où j'ai trempé ma plume. Mais l'impératif réflexif qui forge nos sciences sociales n'interdit nullement bien au contraire de commenter de façon critique et distanciée un collectif découvert en y participant pour sa modeste part.

Commençons d'abord par quelques traits généraux de la situation rapportés par Giordan : 1) la spécialité disciplinaire sort tout récemment d'une période de suspicion en illégitimité scientifique, notamment dans les pays issus des Lumières où la discipline mère semble avoir voulu se détacher de la métaphysique (France, Allemagne, Grande-Bretagne) ; 2) une spécialité trop longtemps associée à la nébuleuse des "sciences religieuses " où l'histoire règne en s'annexant de façon plus ou moins clandestine les services de la théologie ; 3) après un long débat sur la sécularisation supposée du monde occidental, l'intensification des

4. Cette association est née en 1970 de la déconfessionnalisation de l'American Catholic Sociological Society fondée en 1938, à l'instar de ce qui s'est passé en Europe avec la transformation de la Conférence internationale de sociologie religieuse (CISR, fondée en 1948) en Société internationale de sociologie des religions (SISR, 1971). Cf. Sociology and Religions, op. cit.

5. Dans Archives de sciences sociales des religions : P. Lassave, "Entre sociologie et anthropologie. Manuels de poche ", 2008/142, p. 151-167; "The Words of Dictionaries ", 2011/ 156, p. 13-30 ; "Autoportraits de sociologues des religions », 2013/164, p. 191-206. 
échanges entre sociétés au développement inégal réinscrit la question religieuse au cœur de la communautarisation des rapports sociaux; 4) les attentats du World Trade Center en 2001 marquent ce tournant, sans doute déjà amorcé vingt ans plus tôt avec la prise de pouvoir de l'ayatollah Khomeini en Iran.

Faut-il ensuite suivre le plan de croisière proposé qui part de l'Amérique du Nord (Québec, Canada anglais, États-Unis) vers le Sud (Brésil), puis vers l'Europe de haut en bas (Grande-Bretagne, Pays-Bas, Belgique, Allemagne, France, Espagne, Italie, Croatie) pour se terminer au Japon et en Australie? L'empirisme qui présidait au choix des pays n'aurait-il d'égal que celui qui prévaut dans l'ordre de leur présentation ? Signe manifeste d'un défaut de réflexion sur les différences entre cadres nationaux?

Notre commentaire prendra précisément cette matière brute comme point de départ de comparaisons et de mises en relations possibles entre les pays rencontrés. L'analyse secondaire d'un tel corpus concernera aussi bien la forme ou la manière de traiter son cas particulier que le fond où se dessine le paysage conceptuel d'une spécialité du savoir. On ne peut ainsi qu'être frappé par l'écart entre le souci plus ou moins affirmé de la définition des concepts théoriques et des méthodes d'enquête comme c'est le cas pour la France ou l'Allemagne et la simple revue des institutions universitaires avec sa kyrielle de patronymes qui ne parlent qu'aux initiés comme c'est le cas pour les États-Unis. Mais il faut faire attention à ce que ces différences de forme ne viennent pas compromettre la compréhension du fond : comme si la description très empirique des institutions que nous livre Anthony J. Blasi sur le cadre états-unien se trouvait être le reflet d'un manque théorique qui affecterait le savoir en question. L'auteur, ancien président de l'association éditrice et directeur de l'ouvrage, a déjà beaucoup écrit, après d'autres, sur l'origine chrétienne et réformatrice de la sociologie américaine $^{6}$. Il refait cependant ici l'essentiel du chemin qui mène des premières enquêtes de communautés locales jusqu'aux débats théoriques sur la « religion civile » initiés par Robert Bellah en passant par la «construction sociale de la réalité » définie par Peter L. Berger et Thomas Luckmann. Le tableau institutionnel qu'il livre révèle un paysage multipolaire et foisonnant où la sociologie des religions s'inscrit dans un vaste réseau qui relie les facultés théologiques confessionnelles, les Divinity Schools, les observatoires d'opinion associant initiatives privées et publiques, et les chaires et laboratoires universitaires dédiés ça et là à la spécialité. On comprend aisément pourquoi certains de ces derniers ont inventé la théorie du «marché religieux » où la demande latente de réassurance existentielle dans une société oppressante ou bouleversante rencontre l'offre de service symbolique

6. A. J. Blasi, Sociology of Religion in America: A History of a Secular Fascination with Religion, Leiden-Boston, Brill, 2014 ; W. H. Swatos, Faith of the Fathers: Science, Religion and Reform in the Development of Early American Sociology, Bristol, Windham Hall Press, 1985 ; A. J. Vidich, S. M. Lyman, American Sociology: Wordly Rejections of Religion and Their Directions, New Haven, Yale University Press, 1985. 
que les confessions ou les dénominations s'efforcent de délivrer au plus près des personnes.

En d'autres lieux, le destin de la spécialité s’inscrit étroitement dans les méandres de la vie nationale. Au Canada français par exemple, l'Église catholique a été depuis le XVIII ${ }^{\mathrm{e}}$ siècle la matrice identitaire des migrants francophones confrontés à la domination britannique. L'éducation, la santé et même le maintien de la langue lui en sont redevables au-delà de toute séparation légale entre Église et État. C'est alors, comme le montre Martin Meunier de l'Université d'Ottawa, qu'assez naturellement la sociologie a été sollicitée pour comprendre le changement social, notamment le détachement religieux des couches populaires urbaines à l'issue d'un long exode rural. Une note de bas de page suggère à cet égard que la sociologie urbaine de l'École de Chicago des années 1920 était déjà à l'œuvre sur le terrain québécois - on pense aux enquêtes d'Everett Hughes sur la « rencontre de deux mondes » dans un Canada français en transition (1943). Mais l'auteur cale son récit sur le tournant des années 1950, lorsque de jeunes sociologues souvent issus des milieux cléricaux ont emboîté le pas des études monographiques de pratique religieuse initiées en France par Gabriel Le Bras et Fernand Boulard. Une génération de " sociographes pastoraux " d'origine canadienne a même fait souche à l'Université grégorienne de Rome. Mais lorsque le vent sécularisant de la "Révolution tranquille » a tourné à partir des années 1960, l'intérêt pour la chose religieuse s'est progressivement recomposé dans une perspective anthropologique et comparative. Il faut noter à cet égard le rôle important joué par Henri Desroche entrainant à sa suite dans les années 1970 quelques chercheurs français du Groupe de Sociologie des Religions ${ }^{7}$. La perspective "religiologique » instituée à l'Université de Montréal n'a pas tardé à rencontrer les multiples formes de réinvestissements religieux propres aux cycles migratoires qui dynamisent la métropole. Des sociologues québécois sont devenus dès lors experts mondiaux en "accommodements raisonnables » entre revendications communautaires et impératif de coexistence laïque. Coté Canada Anglais, présenté par Michael Wilkinson de l'université de la Trinité en Colombie-Britannique, le cours des choses s'est trouvé en revanche plus étroitement associé à celui des universités américaines, suivant en cela les vagues thématiques de la sécularisation, du marché religieux et de l'individualisation du croire esquissées par Blasi.

À l'instar de la "Belle Province "(Québec), le vaste Brésil témoigne d'un enchevêtrement certain entre les sciences sociales et le destin d'un pays, comme le montre Roberto Motta. Déjà, la devise comtienne "Ordem e Progresso » imprimée sur le drapeau rappelle tout ce que l'État-nation doit au positivisme du premier sociologue. Longtemps, au cours du XXe siècle, la question des élites

7. Voir : J. Palard, «Henri Desroche et ses réseaux québécois. Entre théorie et pratiques maïeuticiennes ", Sociologie et Sociétés, vol. 37, n 2, 2005, p. 32-33. 
a été de savoir pourquoi ce pays de métissage ne réussissait pas à atteindre le stade de développement du grand cousin nord-américain. Sans doute en raison du poids du catholicisme, répondent les sociologues qui ont lu Max Weber. Le capitalisme latifundiaire n'aurait pas trouvé d' "affinité élective » aussi marquante avec l'Église missionnaire que le capitalisme expansionniste avec l'éthique protestante. La philosophie de l'histoire chapeautée par le modèle libéral nordaméricain joue ici de contraste avec la ferveur populaire des cultes les plus baroques associant les divinités des trois continents (Afrique, Amérique, Europe). Dès les années 1930, une sociologie indissociable de l'histoire et de l'ethnologie aide à faire comprendre les transactions entre "Maîtres et esclaves " (célèbre titre de Gilberto Freyre) où le monde des saints détrône parfois celui des patrons en les dépossédant de leur droit exclusif sur la vie et la mort. Dans les années 1950, le français Roger Bastide va jusqu'à s'initier au culte syncrétique du candomblé pour mieux rendre raison de la dynamique entre le «sacré domestique " du jour et le "sacré sauvage » de la nuit. Un temps associés à la "théologie de la libération ", les sociologues œuvrent de fait à la culture altermondialiste par l'étude des mécanismes de circulation entre cultes transnationaux. L'auteur ne précise pas à cet égard les liens noués entre sociologie et ethnologie.

Dans le prolongement de cette évocation brésilienne, la sociologie vient parfois accompagner l'émancipation culturelle d'un pays comme c'est en partie le fait, et à différents niveaux, de l'Espagne, de l'Italie ou de la Croatie. Commençons par le dernier cas de l'ex-Yougoslavie, marginal et moins connu. L'auteur, Sinisa Zrinscak de l'Université de Zagreb, nous fait d'abord part de ses craintes de ne pas être bien compris de ses étudiants de sociologie quand il explique la distinction entre sexe et genre. Ici, les autorités catholiques qui ont repris l'ascendant sur le gouvernement après la période socialiste contestent en effet ouvertement la théorie du genre. Après la période de gel marxiste puis la guerre ethnoreligieuse qui a vidé le pays de sa population musulmane, la sociologie des religions a tout récemment fait son apparition à l'université pour aider la société à prendre sa place dans le concert européen. Une poignée de sociologues s'emploie ainsi à des enquêtes comparatives sur les pays des Balkans dans le cadre de coopérations universitaires à l'échelle de l'Europe orientale.

Réétudiée librement en Espagne après le long tunnel de la dictature franquiste (1939-1975), la sociologie des religions accuse également le coup de son retard et de son parcours erratique, ainsi que le rappelle Mar Griera de l'Université de Barcelone. À l'instar des autres pays d'Europe, l'intelligentsia hispanique au tournant du $\mathrm{XX}^{\mathrm{e}}$ siècle avait pourtant marqué de l'intérêt pour les sciences positives : anarchistes catalans, catholiques sociaux et adeptes du philosophe allemand panenthéiste Karl Krause s’inscrivaient diversement dans ce mouvement ${ }^{8}$.

8. Rappelons que le panenthéisme, terme forgé par Karl Krause (1828), postule que le divin existe, interpénètre la nature et la dépasse ; l'expression se distingue de celle de panthéisme qui tient que le divin est compris tout entier dans l'univers. 
Même écho du monde dans le pays sous la chape franquiste : à l'approche de Vatican II et à rebours du conservatisme d'État, certains religieux se sont ressaisis de la sociologie sur le modèle des enquêtes pastorales initiées par Le Bras et Boulard. La discipline s'est imposée comme instrument de la contestation interne à l'Église. Pourtant, lorsque la démocratie a enfin prévalu et que les départements de sciences sociales se sont mis en place dans les facultés, la sociologie des religions n'a nullement fait partie des priorités. "Autant ouvrir un cours de hongrois à la place de celui d'anglais!" pouvait-on dire à ce sujet. Opposition d'autant plus marquée qu'elle émane parfois de clercs reconvertis à la science, précise la sociologue catalane. Mais les échanges renouvelés avec le monde académique international et la montée en régime de la question religieuse associée aux flux migratoires (en provenance surtout d'Amérique du Sud, du Maroc et de l'exYougoslavie) ont eu progressivement raison de ces appréhensions. Dans les années 2000, environ une centaine de thèses ont été soutenues sur le sujet. Les travaux de Joan Estruch sur l'Opus Dei (1995), de Salvador Giner sur la religion en Espagne (1992) ou de José Casanova sur la "déprivatisation » du religieux dans le monde (1994) sont internationalement connus.

Autre pays où le catholicisme a longtemps pesé sur les structures mentales et institutionnelles, l'Italie entretient une histoire complexe avec la sociologie comme l'indique Giordan. Vers la fin du XIX ${ }^{\mathrm{e}}$ siècle, le positivisme comtien a eu moins d'influence qu'en Espagne et la philosophie idéaliste de l'histoire de Benedetto Croce n'a pas favorisé l'enquête matérielle. Pourtant, diverses voix ont ressaisi le christianisme comme force de civilisation et réserve d'imagination collective (Giuseppe Toniolo, Luigi Sturzo). L'intellectuel marxiste Antonio Gramsci s'est joint à elles pour décrire la diversité des types sociaux se réclamant du catholicisme (populaire, intellectualiste, mystique, etc.). Les péripéties de la dictature mussolinienne ici aussi ont interdit de séjour toute sociologie réflexive. Ce n'est qu'après la Seconde Guerre que la sociologie des religions a pris progressivement ses marques universitaires. Mais dans une certaine division entre la sociographie pastorale façon Boulard et l'anthropologie des religions façon Desroche. À Padoue (Sabino Acquaviva, Vincenzo Pace), à Rome (Franco Ferrarotti, Roberto Cipriani) ou à Trente (Salvatore Abbruzzese), des auteurs se sont progressivement distingués dans les débats internationaux sur la " démagification " du monde, la "religiosité diffuse » ou les nouvelles radicalités. Les manuels de spécialité attestent la productivité de cette génération d'après-guerre sous l'influence du Groupe de Sociologie des Religions français. Sans doute trop centré sur la sous-discipline au sens strict, Giordan manque d'indiquer ses développements connexes ou parallèles, notamment en matière d'histoire et d'anthropologie où les savants italiens se sont pourtant imposés 9. Côté histoire, citons seulement Rafaele Pettazzoni qui dans la lignée prestigieuse de

9. Voir par exemple le manuel de l'historien Giovanni Filoramo (Che cos'è la religione, 2004), récemment traduit en français: Qu'est-ce que la religion? Thèmes, méthodes, problèmes, Paris, Éditions du Cerf, 2007. 
Giambattista Vico a déployé une œuvre conséquente sur les mythes et légendes comparées - il fut notamment conduit à réfuter les thèses du Père Wilhelm Schmidt sur le monothéisme primitif. Côté anthropologie, évoquons également Ernesto de Martino reconnu pour son ethnologie du magico-religieux, notamment le tarentisme (exorcisme de l'araignée dans les Pouilles) ${ }^{10}$.

En suivant cette ligne de contrebande, franchissons dès lors les Alpes pour considérer le troublant cas français où, avec Durkheim, la sociologie s'est instituée autant avec qu'à l'encontre de la religion. Avec, parce que la religion est pour le fondateur de la discipline au cœur du monde social. À l'encontre, parce que la science positive des formes de vie religieuse n'a pu s'instaurer qu'en rupture avec les cadres de pensée issus d'une longue domination cléricale à peine rompue depuis les Lumières. Notre propre contribution reprend le fil de cette histoire en se défiant des faux plats, par exemple le supposé creux de l'entredeux-guerres, ou des continuités invoquées, par exemple la supposée filiation durkheimienne du déjà cité Groupe de sociologie des religions (GSR) des Trente Glorieuses de reconstruction empirique et théorique de la discipline. Incontestablement, Marcel Mauss et ses collaborateurs (Henri Hubert, Maurice Halbwachs) ont su approfondir la fondation durkheimienne en y apportant matière et nuance. L'anthropologie historique des mécanismes sacrificiels permit ainsi d'explorer les canaux réglés de circulation entre le sacré et le profane dont l'opposition radicale est pourtant pour Durkheim au principe de l'identité religieuse. Créé en 1954 au CNRS en rupture avec la sociographie pastorale du catholicisme éclairé, le GSR a d'abord eu recours à la compréhension typologique forgée par Max Weber plutôt qu'à l'explication méthodique des variations concomitantes instaurée par Durkheim. L'herméneutique wébérienne s'est avérée en effet plus appropriée à l'extension du regard à la multiplicité des utopies, dissidences et messianismes de par le monde au-delà du seul paradigme de la sécularisation mis à l'épreuve des colloques internationaux. On a déjà entrevu combien ce groupe et sa revue (Archives de sociologie des religions) avaient joué d'influence pour établir la sous-discipline dans certains pays comme le Québec ou l'Italie (on pourrait rajouter à la liste le Mexique et la Belgique). Outre Le Bras, Desroche et Séguy déjà évoqués, mentionnons : François-André Isambert, analyste de l'efficacité symbolique et de la morale ; Jacques Maître renouvelant l'approche psychohistorique de l'expérience mystique ; Émile Poulat, historien de l'intransigeantisme catholique. Parmi les successeurs nés après-guerre, Danièle Hervieu-Léger a notamment redéfini l'objet religieux comme dispositif d'inscription des sujets dans une lignée qui les légitime. Les années les plus récentes voient les cadres de recherche sociologique suivre la diversification des terrains et des perspectives en dialogue toujours plus étroit avec l'histoire des traditions, l'anthropologie des

10. Voir sur ce dernier point le numéro récent de notre revue (Archives de sciences sociales des religions, 2011, no 161) consacré aux messianismes avec un retour sur l'œuvre de l'ethnologue Vitorio Lanternari, élève de Pettazzoni. 
communautés et la science politique des questions de laïcité. L'histoire postcoloniale de l'islam et son inscription mouvementée dans le libéralisme européen relancent le questionnement sur le "fait religieux ». L'attention renouvelée aux agencements productifs entre êtres et choses, naturels et surnaturels, au principe des modes d'existence religieuse s'appuie en retour sur un certain pragmatisme méthodologique de tonalité américaine. De quoi s'affranchir d'une longue période de débats internationaux et d'esprit fonctionnaliste autour de la «modernité religieuse ». Mais à la différence du prolixe partenaire d'Outre Atlantique, l'offre académique est ici particulièrement circonscrite aux grandes écoles parisiennes lointainement issues de la séparation entre théologie et sciences religieuses au XIX siècle.

C'est sans doute d'Allemagne que provient une autre des principales sources conceptuelles de la sous-discipline. Detlef Pollack, de l'Université de Münster, estime cependant que la reconnaissance académique de la sociologie des religions est tardive : moins d'une dizaine de chaires, mais plus d'une centaine d'auteurs actifs, notamment sur le plan international. Les flux migratoires qui ont suivi la chute du Mur de Berlin, notamment aujourd'hui avec les pays du sud, réactivent également la thématique du fait religieux. Celle-ci a une longue histoire dans la discipline. D'abord et avant la tragédie nazie, les grandes œuvres de Max Weber, de Ernst Troeltsch et de Georg Simmel. Formes de communalisation religieuse, types d'autorité ou de charisme, distinction entre Église, secte et communauté mystique, religiosité manifeste et latente et bien d'autres concepts issus de l'étude des religions historiques marquent de leur empreinte les reprises théoriques de l'après-guerre. L'exil américain de nombre d'auteurs pendant le nazisme fut un premier canal de diffusion des perspectives phénoménologiques. La religion comme « machinerie conceptuelle de maintenance de l'univers » (Peter L. Berger, Thomas Luckmann) ou la dialectique entre « expérience spirituelle et expression religieuse » (Joaquim Wach) vont ainsi innerver les échanges internationaux dans les années 1960-1980. Mais dès l'immédiat après-guerre, la présence des Églises protestantes et catholique dans l'effort de reconstruction du pays suscite également un ensemble d'enquêtes sur les pratiques cultuelles et le changement social sur le modèle initié par Le Bras. À l'instar de la France, la sociologie universitaire s'est affirmée dans la critique du réductionnisme de cette sociographie pastorale. Luckmann a notamment étendu les machineries conceptuelles au-delà des seules pratiques confessionnelles en émettant l'idée de "religion invisible » observable à trois niveaux de transcendance : 1) le mineur (ce que l'on peut imaginer de ce qui se passe derrière la porte, mais qu'on ignore);2) le médian (ce qu'autrui pense réciproquement de moi) ; 3) le majeur (ce que j'aimerais qu'il arrive dans le monde ou la vie). "Sous-système spécifique » dans la théorie de Niklas Luhmann, le religieux n'a pas moins fait l'objet de critiques classiques de Marx à Adorno pour son rôle de substitut illusoire à l'oppression. Plus récemment, le philosophe Jürgen Habermas qui s'inscrit dans cette filiation critique reconnaît pourtant aux traditions religieuses leur apport de sens moral dans un espace 
public pluraliste et en quête de repères. Autant de considérations sur la nature communicationnelle du religieux qui coexistent avec la poursuite des observations quantitatives sur les pratiques et les opinions pour lesquelles les sociologues allemands - tel l'auteur - sont très présents dans les enquêtes internationales ${ }^{11}$.

Présentant le cas de la Belgique, les professeurs Liliane Voyé et Karel Dobbelaere reviennent sur cette autre solidarité de destin entre un microcosme académique local et l'affirmation internationale de la spécialité. Dans ce petit pays bilingue à la socialisation historiquement divisée en trois " piliers » (catholique, socialiste, libéral), c'est d'abord la partie catholique en quête de dépassement de frontières sociales qui s'inscrit après-guerre dans les pas de la sociographie pastorale façon Le Bras-Boulard. Les universités catholiques de Louvain (française et flamande) forment le double foyer de ces enquêtes quantitatives et de leur dépassement sociologique. Toute une génération de prêtres souvent engagés dans le mouvement de réforme de Vatican II rencontre ainsi la sociologie et ses débats méthodologiques opposant le positivisme du chiffre à l'herméneutique sociale. Héritier laïc de ce mouvement, le couple d'auteurs met en relief certaines hautes figures de cette rencontre. Par exemple, celle du "chanoine rouge " François Houtart à l'origine de la revue internationale Social Compass dédiée aux sciences sociales des religions et actif missionnaire en la matière dans les pays en développement. Autre figure, celle de Jean Rémy dont le transfert de la sociographie pastorale vers la sociologie urbaine montre en particulier comment l'héritage de Simmel et de l'École de Chicago permet d'expliquer la mobilité des formes religieuses et des aires morales. Dobbelaere, théoricien de la sécularisation très actif dans la société internationale de sociologie des religions, s'interroge pour finir sur le risque d'épuisement des catégories de l'entendement sociologique face à la formulation communautariste des questions de coexistence sociale (division entre Wallons et Flamands, enclavements ethno-religieux). L'anthropologie et la science politique lui semblent à cet égard devoir prendre le relais.

Si la Belgique s'est construite au début du XIX ${ }^{\mathrm{e}}$ siècle contre la domination protestante hollandaise à l'origine des Pays-Bas actuels, ceux-ci s'étaient auparavant construits au XVII ${ }^{\mathrm{e}}$ siècle contre l'influence de Rome. Dans les deux cas pourtant un même système de "pilarisation » de la société prévaut, avec comme en Allemagne une fonctionnarisation des clercs. À l'instar des États-Unis notamment, les Religious Studies s'en trouvent réparties entre facultés «libres » ou laïques et facultés confessionnelles. En Belgique, le pilier anticlérical de la librepensée est ainsi à l'origine de l'Université Libre de Bruxelles, avec son actif laboratoire de sciences sociales du religieux qui se distingue des sciences religieuses de l'Université Catholique de Louvain. Aux Pays-Bas, les flux démographiques ont fait qu'au $\mathrm{XX}^{\mathrm{e}}$ siècle, la minorité catholique a pris numériquement le dessus

11. Voir également le dossier coordonné par Hubert Knoblauch et Anne-Sophie Lamine, "Postérités allemandes ", in Archives de sciences sociales des religions, 167/2014. 
sur la majorité protestante, même si depuis les années 1960 l'influence de ces deux piliers cède du terrain aux nouvelles minorités dont la dernière en date est la musulmane. Enseignants-chercheurs au double cursus de théologie catholique et de sociologie, Kees de Groot et Erik Sengers retracent le parcours désormais convenu de la spécialité disciplinaire depuis l'après-guerre : sociographie pastorale, puis théories de la sécularisation, puis recompositions post-séculières et communautaires qui posent la question de la pertinence de l'appareil sociologique. Ici également ce dernier semble survivre à la crise de perspectives à travers la défense corporative de son savoir en s'appuyant notamment sur la coopération transfrontière.

Si les histoires belges et néerlandaises se centrent principalement sur ces évolutions d'après-guerre, celle du cas britannique, retracée par Rebecca Catto de l'Université de Coventry, remonte plus heureusement dans le temps. Elle montre ainsi comment dans la société victorienne du XIX ${ }^{\mathrm{e}}$ siècle la science des religions suit une tradition naturaliste marquante (David Hume) et se ressource dans le positivisme d'Auguste Comte, le libéralisme de Herbert Spencer et le romantisme de John Ruskin. L'empire colonial explique sans doute l'attention portée aux sociétés dites primitives et aux mondes orientaux. D'où les perspectives évolutionnistes à trois temps : animisme, polythéisme, monothéisme (Edward Tylor); magie, religion, science (James Frazer). À l'approche du Xx siècle, la mythologie comparée et la question totémique voisinent ainsi sans dialogue avec les enquêtes philanthropiques sur la condition ouvrière en métropole, prémices de la discipline sociologique. Il faut attendre l'après-Seconde Guerre pour que la thématique religieuse s'inscrive dans la discipline qui se détache alors des savoirs connexes tels que la philosophie, l'anthropologie, l'histoire ou la théologie. Mais rien n'est dit sur ce processus même de distinction des perspectives. En effet, le récit embraye aussitôt sur les phases et les auteurs majeurs de la sous-discipline sociologique au sens étroit du terme. Moment d'enquêtes empiriques sur les pratiques et l'organisation des paroisses urbaines, anglicanes principalement, dans la reconstruction des années 1950. Puis attention portée aux nouvelles expressions religieuses et notamment aux « dérives sectaires » et " manipulations mentales » dans les années 1970 dites de sécularisation. Enfin, à partir des années 1990, l'accent mis sur les processus de communautarisation liés aux flux migratoires. À l'instar des pionniers français d'après-guerre, on peut ici citer Bryan Wilson (travaux sur les sectes), David Martin (critique de la sécularisation), Roland Robertson (théories de la globalisation) ou William Pickering (relance des études durkheimiennes). Leurs élèves, aujourd'hui en passe de transmettre l'héritage, se sont distingués par des travaux et expériences originaux (par exemple, Grace Davie et son célèbre "Believing without belonging », Eileen Baker et Jim Beckford et leur recherche-action sur les "nouveaux mouvements religieux »). Également répartis entre départements théologiques et de sciences sociales à l'instar des universités américaines, ces auteurs bénéficient par ailleurs de leur avantage linguistique dans les instances internationales. 
Influence que l'on retrouve naturellement à l'autre bout de l'hémisphère dans l'ex-dominion australien. Terre coloniale où au tournant du siècle dernier l'ethnographie des aborigènes (Francis Guillen, Walter Spencer, Carl Strehlov) fournit matière aux débats sur le totémisme et à la théorie durkheimienne de la religion. Mais ici encore la présentation de la sous-discipline sociologique fait l'impasse sur cette histoire. Le groupe d'enseignants-chercheurs qui s'y attelle (Gary Bouma, Douglas Ezzy, Anna Halafoff, Adam Possamai) s'en tient à une sorte de manifeste sur l'utilité de la connaissance du fait religieux dans une société « interculturelle» de plus en plus traversée de tensions entre vagues migrantes. La majorité chrétienne, protestante principalement en ses multiples dénominations, cède en effet du terrain depuis les années 1980 aux populations musulmanes (Indonésie) et bouddhistes (Asie). La " révolution conservatrice » du début des années 2000 semble avoir en vain résisté aux revendications d'égalité de droit entre « communautés ». D'où l'utilité de comprendre les transformations sociales de la «mosaïque ». Tâche à laquelle une poignée de sociologues s'emploient, en lien actif avec les organisations non gouvernementales telles l'UNESCO.

Last but not least, le Japon, dernier pays visité, donne tous les signes d'une égale extraversion intellectuelle. Mais, comme le montre Michel K. Roemer, spécialiste américain de la culture nipponne, l'intérêt des universitaires japonais pour les sciences sociales se traduit pour leurs homologues occidentaux par la remise en cause de leurs catégories de pensée. Bien qu'à travers Marx, Durkheim et Weber la sociologie des religions n'ait pas été absente des lectures universitaires dès le début du siècle dernier, ce sont surtout les études ethnographiques des traditions insulaires qui ont longtemps fait office de sciences religieuses. L'attention à la sociologie des religions s'est précisée à partir des années 1970 tout en faisant l'objet d'ajustements sémantiques fondamentaux. Îlot de syncrétisme entre bouddhisme, confucianisme et shintoïsme, le pays du Soleil-Levant ne trouve guère que le foyer familial comme équivalent des Églises occidentales. Tout individu peut selon les moments honorer de façon alternative ses engagements envers tel culte ou mode de faire. La croyance s'y distingue mal de l'attitude ordinaire de même qu'il est bien difficile d'établir un distinguo entre religion et tradition. Les théories de la sécularisation y ont difficilement prise dès lors que les signes de religiosité, comme dirait Simmel, sont durablement inscrits dans cette sorte de religion civile entretenue par l'équilibre des dettes symboliques réciproques entre individus. Certes les sociologues des religions se sont faits les analystes minutieux des nouveaux mouvements religieux et des dérives sectaires à l'instar de leurs correspondants occidentaux (mouvements Sokka Gokai ou secte Aum et son attentat au gaz sarin dans le métro de Tokyo en 1995), mais l'auteur insiste finalement sur le laboratoire des catégories de pensée que constitue le Japon pour ses partenaires occidentaux.

Au terme de ce petit tour du monde, revenons sur certains points ou questions transversales. Et d'abord sur les angles morts de l'entreprise. Outre l'absence 
mentionnée de nombre de continents, les différences d'appréhension des cas (catalogue institutionnel pour les États-Unis, mise en situation des concepts pour l'Allemagne ou la France) ne sont pas explicitées dans la présentation de l'ensemble au même titre d'ailleurs que la discrétion qui entoure le processus de sélection de l'échantillon. Ces histoires nationales limitent pour l'essentiel leur champ temporel et matériel d'investigation à la dénomination stricte ou institutionnelle de la sous-discipline. D'où le peu d'éléments à relever sur les variations nationales de construction du savoir sociologique avant son autodéfinition. Les cas britannique et italien sont intéressants à cet égard dans la mesure où l'anthropologie culturelle pour le premier et l'histoire des religions pour le second préexistaient chacune à la formation de la spécialité disciplinaire après-guerre. Mais comme on l'a indiqué, la refondation sociologique sur la base empirique des terrains contemporains tend à faire oublier les solidarités natives entre sciences sociales du religieux. D'où la difficulté à se représenter un corps de connaissance aux frontières variables et construit comme un nœud de réseau entre perspectives conjointes. L'idée se confirme dès lors que ce recueil est bien le produit ou le reflet parlant d'une prise corporative du savoir au sein du jeu universitaire de places entre disciplines et spécialités aux contours aussi figés que mouvants.

De la sorte, les pays où la sociologie des religions est présentée comme une création récente se retrouvent dans un schème d'évolution semblable : d'abord la comptabilité paradoxale des cultes au moment où les pratiques s'individualisent et les attachements se fluidifient, puis des réflexions sur la nature et le devenir de la sécularisation, enfin des explorations sur les formes de religiosités résiduelles ou actives, de plus en plus politiquement instrumentalisées. À terme, les observateurs accusent le coup, comme c'est le cas pour la Belgique, d'une difficulté à renouveler les paradigmes et les postes dans le cadre d'une spécialité trop étroite. Pour la plupart et notamment pour les nouveaux entrants comme l'Australie ou la Croatie, l'espace des relations et des échanges internationaux déjoue la menace d'enfermement et pallie l'absence de tradition. En retour, les pays de plus longue tradition sociologique risquent de se satisfaire de leurs glorieux états de service théorique au détriment d'une acculturation mondiale qui renouvellerait les frontières du savoir.

Les flux de traductions mériteraient à cet égard d'être étudiés dans leur évolution. On sait ainsi que les œuvres fondatrices de Weber et de Simmel sont souvent passées en langue anglaise avant de se retrouver en français, italien ou espagnol. De même, les Formes élémentaires de la vie religieuse de Durkheim ont-elles transité en anglais avant de se retrouver en chinois ${ }^{12}$. Ce travail de suivi reste à faire pour y voir plus clair dans les inégalités d'échange intellectuel entre

12. L. Roulleau-Berger, Zhengai Liu, "La théorie de la religion de Durkheim et la sociologie chinoise ", Archives de sciences sociales des religions, 2012, n 159, p. 135-151. 
sociétés ${ }^{13}$. Le présent recueil indique en creux quelques influences, transferts voire tutelles d'un pays à l'autre. Le modèle d'enquête sur les pratiques religieuses en milieu catholique et rural mis en place par Gabriel Le Bras dans les années 1930, puis étendu après-guerre à de multiples paroisses urbaines par le chanoine Fernand Boulard et ses «enquêteurs du dimanche » ${ }^{14}$, semble ainsi avoir essaimé à travers l'Europe et au-delà. On s'y réfère en effet dans les cas de la Belgique, de l'Italie, de l'Espagne, de l'Allemagne, de la Grande-Bretagne, des Pays-Bas et du Québec. Ces dynamiques de coopération internationale restent à préciser. La Conférence internationale de sociologie religieuse (CISR) créée en 1948 à l'université catholique de Louvain par des clercs faisant vœu de sociologie fut le lieu principal de cette coopération. Émile Poulat et Karel Dobbelaere en ont retracé les évolutions, notamment la transformation séculière de l'association dans les années 1970 qui se solda par la création en 1989 de la Société internationale de sociologie des religions (SISR) sans plus aucune référence à quelque horizon confessionnel ${ }^{15}$. Il reste également à retracer le rôle qu'a pu jouer le Groupe de sociologie des religions français (GSR) dans ce mouvement de déconfessionnalisation de la science. On l'a entrevu à propos de l'université de Sherbrooke au Québec, plaque tournante de la coopération franco-canadienne en la matière durant les années 1970. Il faudrait sans doute étendre l'examen des voies d'échanges, de transferts ou d'influence, par exemple entre le GSR et les sociologues belges, italiens ou brésiliens, sans oublier le continent africain, grand absent du recueil.

Si cet assortiment bien limité d'histoires nationales part du principe que tout savoir, aussi universel soit-il dans sa vocation, n'en est pas moins le produit d'une formation sociale particulière, il a au moins le mérite de laisser entrevoir aussi les rôles politiques variables que peut jouer la sociologie suivant les contextes. Il faudrait à cet égard revenir plus précisément sur les processus successifs qui ont amené le positivisme comtien à figurer comme emblème de la constitution brésilienne, de même que l'on a pu décliner la sociologie durkheimienne comme institutrice de la Troisième République avec en parallèle l'œuvre wébérienne pour la République de Weimar ${ }^{16}$. À une échelle, certes plus modeste, se jouent le rôle et le destin d' "écoles » ou de groupes liés à notre spécialité

13. Sur cet échange inégal, voir plus globalement: Traduire la littérature et les sciences bumaines. Conditions et obstacles (G. Sapiro, dir.), Paris, Ministère de la Culture et de la Communication, 2012.

14. A. Chenu, "Les enquêteurs du dimanche. Revisiter les statistiques françaises de pratique du catholicisme (1930-1980) ", Histoire \& Mesure, 2011, no 26, p. 177-221.

15. É. Poulat, "La CISR, de la fondation à la mutation : réflexions sur une trajectoire et ses enjeux ", Social Compass, 1990, n 37, p. 11-33 ; K. Dobbelaere, "The International Society for the Sociology of Religion (SISR): The Last Decade of the 20th Century ", in Sociology and Religions. An Ambiguous Relationship, op. cit., p. 90-100.

16. P. Arbousse-Bastide, Le positivisme politique et religieux an Brésil, Turnhout, Brépols, 2010 ; H. Tyrell, «Religion et politique - Max Weber et Émile Durkheim », Trivium, 2013, $\mathrm{n}^{\circ}$ 13. [En ligne : trivium.revues.org]. 
thématique. Sous réserve d'inventaire dans les pays concernés, il semble donc que le travail de déconfessionnalisation des catégories de pensée opéré par le GSR ait convergé avec le processus de relativisation historique des traditions chrétiennes qui a traversé le concile de Vatican II. Toute Église n'est plus dès lors à concevoir dans sa vocation universelle, mais dans les forces sociales plurielles et contradictoires qui la traversent et menacent son intégrité. La supposée « religion populaire » s'est par exemple trouvée l'otage d'un débat postconciliaire mettant aux prises ses pourfendeurs du côté de la réforme (abandon de la messe en latin, etc.) et ses défenseurs du côté de l'alliance objective entre courants traditionalistes et populistes. On doit alors aux sociologues la mise en scène académique d'un tel débat conduisant de la sorte la théologie à se dissoudre dans les arcanes de la logique sociale ${ }^{17}$.

Mutatis mutandis, quelques décennies plus tard, les sociologues se voient dans le même pays accusés par un Premier ministre d'excuser le terrorisme d'obédience djihadiste en allant lui trouver des raisons dans la relégation sociale ou dans la revanche postcoloniale. "Expliquer n'est pas excuser " répliquent-ils aussitôt comme ils avaient pu le faire une décennie auparavant à propos des dérives sectaires ${ }^{18}$. Mais plus largement, la question se pose de la nature religieuse ou pas de la référence à l'islam dans l'explication de crimes de masse ou d'actes quotidiens qui, tel le voilement ostensif et plus ou moins intégral du corps féminin, remettraient en cause certains principes de vie républicaine ${ }^{19}$. Autant d'épreuves qui poussent la sociologie des religions à se faire actrice publique, notamment lorsqu'elle tend par nature à faire prendre au sérieux la spécificité religieuse des engagements de soi les plus meurtriers en les reliant par degrés aux pensées et gestes ordinaires sans omettre pour autant les conditions postcoloniales ou de relégation sociale qui définissent la situation. Nous sortons là certes du cadre du collectif international et disciplinaire en question. Mais l'actualité française sous état d'urgence invite à la réflexion sur ce que les savoirs empiriques et objectifs sur les phénomènes religieux peuvent faire au gouvernement des sociétés. Ultime effet de notre lecture qui mérite quelques précisions.

Si l'on s'en tient par exemple aux débats récents suscités dans la presse, trois lignes de front apparaissent à cet égard ${ }^{20}$ : d'abord, certains islamologues semblent devoir dénier à la sociologie la possibilité de comprendre ce qui se joue au présent sans passer par la case théologique dans la langue même de ses arcanes ; ensuite, certains sociologues des religions disent combien la reconnaissance de l'objet religieux n'est jamais allée de soi dans une tradition épistémique

17. F.-A. Isambert, «Le sociologue, le prêtre et le fidèle ", in La sagesse et le désordre. France 1980 (H. Mendras, dir.), Paris, Gallimard, 1980, p. 219-246.

18. B. Lahire, Pour la sociologie. Et pour en finir avec la «culture de l'excuse», Paris, La Découverte, coll. «Cahiers libres », 2016.

19. J. Birnbaum, Un silence religieux. La ganche face au diihadisme, Paris, Éditions du Seuil, 2016.

20. M. Rousset, "Les yeux dans les dieux. Enquête », Le Monde, 19 mars 2016. 
née précisément de la rupture avec toute métaphysique et prophétisant le dépassement historique du sentiment religieux; enfin, d'autres invoquent autant Durkheim que Weber pour considérer que le destin de leur discipline se joue précisément dans sa capacité à mettre en relation l'expérience existentielle et son expression religieuse dans la multiplicité et la mobilité de ses formes sociales. Plus que jamais, la sociologie des religions ne peut donc survivre sans intégrer les savoirs liés à la vie religieuse (phénoménologie, ethnologie, psychologie) et à sa traduction logico-narrative (philologie, théologie, histoire). D'où d'ailleurs l'appellation de "science sociale du religieux » qui semble devoir remplacer le terme de "sociologie des religions" lequel avait voulu pourtant prendre ses distances avec la "sociologie religieuse » trop suspecte de connivence avec la pastorale chrétienne. Mais aux figures dédoublées du clerc et du sociologue d'hier s'adjoignent au présent les figures circulantes du ministre, de l'islamologue et du politologue. Le dialogue à fronts renversés entre la « radicalisation de l'islam » et "l'islamisation de la radicalité », bien que largement surfait par le journalisme, laisserait entendre que la sociologie n'a pas pu trouver sa place dans ce concert ${ }^{21}$. La dissolution des essences et la déconstruction de la pensée binaire qui fait la marque de fabrique de la tradition critique en sociologie ont sans doute du retard à rattraper sur le terrain de l'islam si tant est que le jeu soit possible. Entre l'orientalisme du passé colonial et les controverses d'aujourd'hui autour du radicalisme islamiste en passant par les enquêtes sur la condition migrante qui ne savent comment intégrer à leur questionnaire la variable religieuse, une généalogie du savoir s’impose. Celle-ci passe non seulement par le conflit des disciplines et écoles, mais aussi par les lieux divers de définition des problèmes et d'élaboration de normes, tels les think tanks et autres commissions des sages $^{22}$. Où le discours critique des sciences sociales se fait bien difficilement entendre dans un concert d'élite à visée normative et à résonance médiatique préférant le récit édifiant et l'anecdote parlante au labeur de la preuve et à la pensée du complexe ou de l'équivoque ${ }^{23}$.

Pierre LASSAVE

Centre d'études en sciences sociales du religieux (CéSor) UMR 8216 (CNRS-EHESS)

lassave@ehess.fr

21. G. Kepel, «Radicalisation et islamophobie : le roi est nu », Libération, 14 mars 2016 ; M. Lemonnier, "Islamisation de la radicalité ou radicalisation de l'islam ", Entretien avec O. Roy, L'Obs, 7 avril 2016.

22. Voir : "Le consensus des experts " (N. Marzouki, coord.), Archives de sciences sociales des religions, 2011, $\mathrm{n}^{\circ} 155$, p. 11-100. Sur les imbrications d'espaces publics en Europe: N. Göle, Musulmans au quotidien. Une enquête sur les controverses autour de l'islam, Paris, La Découverte, coll. "Cahiers libres », 2015.

23. Sur la construction du "problème musulman » en France et la naissance d'un « champ de la laïcité » où le récit anecdotique fait souvent office de preuve, disqualifiant de la sorte le raisonnement critique des sciences sociales : J. Beaugé, A. Hajjat, «Élites françaises et construction du "problème musulman”. Le cas du Haut Conseil à l'Intégration (1989-2012) ", Sociologie, 2014-1, vol. 5, p. 31-59. 
Annexe

\section{Contents}

- William H. Swatos, Preface: Finding Ourselves

- Giuseppe Giordan, Introduction: How to Tell the Story of the History of the Sociology of Religion

- E.-Martin Meunier, Sociology of Religion in French Canada: Vicisitudinous Relations between Autonomous Knowledge and Institutional Dependence

- Michael Wilkinson, The Historical Development of the Sociology of Religion in English-Speaking Canada

- Anthony J. Blasi, Sociology of Religion in the United States

- Roberto Motta, Social Scientists and Disenchanters: Some Basic Themes of Brazilian Sociology of Religion

- Rebecca Catto, Sociology of Religion in Great Britain: Interdisciplinarity and Gradual Diversification

- Kees de Groot and Erik Sengers, Sociology of Religion in Netherlands

- Karel Dobbelaere and Liliane Voyé, Sociology of Religion in Belgium: Fron Institutional Reference to a Sociological Analysis of Society

- Detlef Pollack, The Sociology of Religion in Germany since 1945

- Pierre Lassave, France: Refounding the Sociology of Religion from Émile Durkheim to the Groupe de Sociologie des Religions and Beyond

- Mar Griera, Politics, Religion and Sociology in Spain: The History of a Discipline

- Giuseppe Giordan, The Sociology of Religion in Italy

- Sinisa Zrinscak, Society Under Siege: The Peculiar Story of the Development of the Sociology of Religion in Croatia

- Michael K. Roemer, A History of the Sociology of Religion in Japan

- Gary D. Bouma, Douglas Ezzy, Anna Halafoff, and Adam Possamai, Sociology of Religion in Australia 